\title{
28 Research Square

\section{SUVmax Reduction Predicts Long-Term Survival in Patients of Non-pCR Both in the Tumor and Lymph Nodes after Neoadjuvant Chemoradiotherapy in Esophageal Squamous Cell Carcinoma}

Yushi Nagaki ( $\nabla$ standoffdatta10@yahoo.co.jp )

Akita University Hospital

\section{Satoru Motoyama}

Akita University Hospital

Yusuke Sato

Akita University Hospital

Akiyuki Wakita

Akita University Hospital

Hiromu Fujita

Akita University Hspital

Yoshihiro Sasaki

Akita University Hospital

Kazuhiro Imai

Akita University Hospital

Yoshihiro Minamiya

Akita University Hospital

\section{Research}

Keywords: ESCC, NACRT, non-pCR, SUVmax

Posted Date: January 21st, 2021

DOI: https://doi.org/10.21203/rs.3.rs-150878/v1

License: (c) (i) This work is licensed under a Creative Commons Attribution 4.0 International License.

Read Full License

Version of Record: A version of this preprint was published at World Journal of Surgical Oncology on April 9th, 2021. See the published version at https://doi.org/10.1186/s12957-021-02208-3. 


\section{Abstract}

Background: A pathological complete response (pCR) after neoadjuvant chemoradiotherapy (NACRT) ensures long-term survival in esophageal squamous cell carcinoma (ESCC) patients following esophagectomy, but pCR patients are a minority. The aim here was to identify prognostic factors in patients with non-pCR ESCC after NACRT.

Methods: This is a retrospective study. Investigated were 5-year overall survival (OS), disease-specific survival (DSS), and relapse-free survival (RFS) among non-pCR ESCC patients divided into pTONO, primary site $p C R(p T O N+)$, lymph node $p C R(p T+N 0)$, and non-pCR in both the tumor and lymph nodes

$(\mathrm{pT}+\mathrm{N}+)$ subgroups after NACRT and esophagectomy. Focusing on the SUV max reduction rate in the primary tumor in 88 patients who underwent FDG-PET before and after NACRT, we used univariate and multivariate Cox proportional hazard models to identify prognostic factors.

Results: Although there were no significant survival differences among non-pCR ESCC patients with pTON+, pT+N0, or pT+N+, and survival rate among p $+\mathrm{N}+$ patients was the poorest. After setting a $60 \%$ cutoff for the SUV $\mathrm{max}_{\max }$ reduction rate in the tumor, RFS curves for non-pCR patients significantly differed between patients above the cutoff and those below it. For $\mathrm{pT}+\mathrm{N}+$ patients, the SUV $\mathrm{max}_{\text {max }}$ reduction rate $(<60 \%$ vs $\geq 60 \%$ ) was an independent prognostic factor of OS, DSS, and RFS.

Conclusion: Because ESCC patients with SUV max $_{\text {max }}$ reduction rates of $\angle 60 \%$ in the tumor after NACRT and categorized as $\mathrm{pT}+\mathrm{N}+$ after NACRT had significantly poorer prognoses, even after esophagectomy, a change in treatment strategy may be an option to improve survival.

\section{Background}

Esophageal cancer, particularly at advanced stages, is a highly aggressive tumor with a poor prognosis. Esophageal squamous cell carcinoma (ESCC) accounts for approximately $90 \%$ of all esophageal cancer cases worldwide and is the most common histological subtype in Asia, Africa and South America. ${ }^{1}$ Because many ESCC patients are diagnosed with locally advanced tumors with lymph node metastasis, multimodal treatment combining surgery with chemotherapy and/or radiotherapy has been introduced to improve patients prognosis. ${ }^{1}$ In this effort, several clinical trials have shown the efficacy of neoadjuvant chemotherapy (NACT) and neoadjuvant chemoradiotherapy (NACRT). ${ }^{2-9}$ NACRT leads to marked downstaging of ESCC tumors, and a pathological complete response (pCR) to NACRT is a key factor contributing to survival in patients with esophageal cancer. ${ }^{10}$ On the other hand, little has been reported about the prognosis of non-pCR patients, who account for the majority ESCC patients treated with NACRT. Specifically, few studies have focused on the difference in prognosis between primary tumor pCR $(\mathrm{pTON}+)$, lymph node pCR ( $\mathrm{pT}+\mathrm{N} 0)$, and non-pCR of both primary tumor and lymph node $(\mathrm{pT}+\mathrm{N}+)$ in patients with ESCC. 
${ }^{18} \mathrm{~F}$-fluorodeoxyglucose-positron emission tomography (FDG-PET) is a functional imaging examination that has been used to assist in the diagnosis of cancer, particularly diagnosis of metastasis and recurrence. The maximum standardized uptake value (SUV $\mathrm{max}_{\text {ax }}$ ), a parameter of FDGPET, is a measure of the highest FDG uptake within a region of interest. It has been reported that the SUV $V_{\max }$ reduction rate in the primary tumor after NACRT is a valuable predictor of pathological therapeutic effect and the survival of patients of esophageal cancer. ${ }^{11}$ In the present study, we used relative changes in the SUV $V_{\text {max }}$ after NACRT to investigate the key factors contributing to long-term survival in non-pCR patients.

\section{Methods}

\section{Patients}

Included in this retrospective study were 115 consecutive patients with confirmed thoracic ESCC with lymph node metastasis and without distant metastasis. But patients with supraclavicular lymph node metastasis were included, because supraclavicular lymph nodes were considered to be locoregional node metastasis in Japan. The patients were treated with NACRT followed by esophagectomy at Akita University Hospital between 2009 and 2017. NACRT was recommended for patients with either a clinical T3-4 primary tumor or regional lymph node metastasis, and with an Eastern Cooperative Oncology Group performance status (ECOG PS) of $0-1$. Clinical staging was determined according to the TNM classification of the UICC (8th edition) ${ }^{12}$ based on upper gastrointestinal endoscopy, esophagography, contrast-enhanced CT, and FDG-PET.

\section{Neoadjuvant Chemoradiotherapy (nacrt)}

The NACRT protocol entailed radiotherapy (40.0-41.4 Gy in 20-23 fractions) with two courses of combined chemotherapy composed of 5 -fluorouracil (5-FU) $800 \mathrm{mg} / \mathrm{m}^{2} /$ day on days $1-5$ plus cisplatin (FP) or nedaplatin (FGP) $80 \mathrm{mg} / \mathrm{m}^{2} /$ day on day 1 . High-energy X-rays (10 MV) were used for the radiotherapy. All patients underwent 3-dimensional radiotherapy planning, and the radiotherapy target was set around the gross tumor volume and metastatic lymph nodes.

\section{${ }^{18}$ F-fluorodeoxyglucose-positron emission tomography (FDG-PET)}

FDG-PET was standard to perform before NACRT and then 3-4 weeks after NACRT, before surgery. However, since it was performed only on outpatients, FDG-PET was not performed on hospitalized patients with severe esophageal stenosis due to esophageal cancer. The FDG-PET was performed with one scanner and procedure was standardized by radiologist. Patients fasted for at least 4 hours before receiving an intravenous injection of $185 \mathrm{MBq} / \mathrm{kg}$ FDG (FDGscan; Nihon Medi-Physics) and then rested for 1 hour before scanning. All images were acquired using a combined PET/CT scanner (Discovery ST Elite 16; GE Healthcare). Low-dose CT images were acquired using a 16-detector row scanner with the following parameters: $120 \mathrm{kV}$, auto $\mathrm{mA}$ (noise index 25.0), $0.6 \mathrm{~s}$ tube rotation, $3.75 \mathrm{~mm}$ section thickness, 
$512 \times 512$ matrix, and $70 \mathrm{~cm}$ field of view. The CT scan was acquired while the patient engaged in shallow breathing and covered an area from the upper thigh to the base of the skull. The SUV $\mathrm{max}_{\text {mas }}$ established for each patient by drawing regions of interest around the primary tumor on attenuation-corrected FDGPET images and then calculating the value using software integrated into the PET/CT scanner. The formula used for the calculation was as follows: $\left.\operatorname{SUV}_{\max }=(\mathrm{C}[\mathrm{mCi} / \mathrm{ml}] / I \mathrm{IDCi}]\right) /$ body weight, where $\mathrm{C}$ was the activity at a pixel within the tissue identified as a region of interest, and ID was the injected dose per kilogram of body weight. The SUV max $_{\text {max }}$ reduction rate was calculated as follows: $\left\{\left(\right.\right.$ baseline $\left.S U V_{\max }\right)-$ $\left(S U V_{\max }\right.$ after NACRT)\} / (baseline SUV $\left.\max \right) \times 100 \%$.

\section{Surgery}

Esophagectomy was scheduled to be performed more than 3 weeks after completing NACRT, by which time patients had no treatment-related adverse events worse than grade 2 according to the Common Terminology Criteria for Adverse Events (CTCAE) Version 4.0. ${ }^{13}$ Esophagectomy under right thoracotomy or thoracoscopic esophagectomy with extended 3-field lymph node dissection was performed.

\section{Pathological Analysis}

The surgically resected thoracic ESCC specimens were subjected to routine pathological examination. We divided the patients into pCR and non-pCR groups based on the pathological diagnosis. Additionally, nonpCR patients were subdivided into $\mathrm{pTON}+\mathrm{pT}+\mathrm{N} 0$, or $\mathrm{pT}+\mathrm{N}+$.

\section{Statistical analysis}

Continuous variables are presented as the median (range: minimum-maximum). Differences between groups were analyzed using the chi-square test to compare non-continuous variables and the KruskalWallis test for continuous variables. Overall survival (OS), disease-specific survival (DSS), and relapsefree survival (RFS) curves were estimated using the Kaplan-Meier method and were compared using the log-rank test. The univariate Cox proportional hazard model was used to assess associations between clinicopathological characteristics and survival. Multivariate analysis was used to adjust for the effects of other variables. All statistical analyses were performed using JMP14 (SAS Institute). All the P values were reported as two-sided with a significance level of 0.05 .

\section{Results}

\section{Patients and tumor characteristics}

Of 115 ESCC patients enrolled in this retrospective study, chemotherapy consisting of FP was used for 43 (37\%) patients, while FGP was used for 73 (63\%) patients. The interval between NACRT and surgery was $40(21-95)$ days. Surgery entailed complete esophageal resection (R0) in all patients. The median 
number of dissected lymph nodes was 49 (8-97). We categorized $26(22 \%)$ patients as pTON0, 10 (8\%) as pTON+, $40(34 \%)$ as pT $+\mathrm{N} 0$, and $40(34 \%)$ as pT $+\mathrm{N}+$. Their characteristics are shown in Table 1 . The median length of follow-up for censored cases was 55.5 (18-60) months after surgery. Forty-six (40\%) patients experienced recurrences during the observation period. Of those, $37(80 \%)$ recurrences were distant metastasis, and 9 (20\%) were local metastasis. 
Table 1

Clinicopathological features of the ESCC patients enrolled in this retrospective study

\begin{tabular}{|c|c|c|c|c|c|}
\hline \multirow[t]{3}{*}{ Characteristic, n (\%) } & \multicolumn{2}{|l|}{ PCR } & \multicolumn{2}{|l|}{ non pCR } & \multirow[t]{3}{*}{$P$} \\
\hline & pTONO & pTON1-3 & pT1-4N0 & pT1-4N1-3 & \\
\hline & $n=25$ & $n=10$ & $n=40$ & $n=40$ & \\
\hline Median age (range) & $\begin{array}{l}63(44- \\
77)\end{array}$ & $\begin{array}{l}65(53- \\
75)\end{array}$ & $\begin{array}{l}66(43- \\
74)\end{array}$ & $\begin{array}{l}63(41- \\
75)\end{array}$ & 0.2402 \\
\hline Gender & & & & & 0.7178 \\
\hline Female & $5(20.0)$ & $1(10.0)$ & $8(20.0)$ & $5(12.5)$ & \\
\hline Male & $20(80.0)$ & $9(90.0)$ & $32(80.0)$ & $35(87.5)$ & \\
\hline Tumor location & & & & & 0.8228 \\
\hline Upper & $5(20.0)$ & $2(20.0)$ & $8(20.0)$ & $9(22.5)$ & \\
\hline Middle & $9(36.0)$ & $4(40.0)$ & $18(45.0)$ & $21(52.5)$ & \\
\hline Lower & $11(44.0)$ & $4(40.0)$ & $14(35.0)$ & $10(25.0)$ & \\
\hline Tumor differentiation & & & & & 0.5425 \\
\hline Not poorly & $20(80.0)$ & $8(80.0)$ & $36(90.0)$ & $36(90.0)$ & \\
\hline Poorly & $5(20.0)$ & $2(20.0)$ & $4(10.0)$ & $4(10.0)$ & \\
\hline Depth of invasion (cT) & & & & & 0.0567 \\
\hline T1-2 & $5(20.0)$ & $3(30.0)$ & $2(5.0)$ & $3(7.5)$ & \\
\hline T3-4 & $20(80.0)$ & $7(70.0)$ & $38(95.0)$ & $37(92.5)$ & \\
\hline $\begin{array}{l}\text { Lymph node metastasis } \\
\text { (cN) }\end{array}$ & & & & & 0.5858 \\
\hline N1 & $18(72.0)$ & $8(80.0)$ & $26(65.0)$ & $24(60.0)$ & \\
\hline N2-3 & $7(28.0)$ & $2(20.0)$ & $14(35.0)$ & $16(40.0)$ & \\
\hline Distant metastasis (cM) & & & & & 0.2138 \\
\hline MO & $19(76.0)$ & $10(100)$ & $36(90.0)$ & $35(87.5)$ & \\
\hline M1 ( Lymph node) & $6(24.0)$ & $0(0)$ & $4(10.0)$ & $5(12.5)$ & \\
\hline Clinical stage(cStage) & & & & & 0.1051 \\
\hline
\end{tabular}




\begin{tabular}{|c|c|c|c|c|c|}
\hline \multirow[t]{3}{*}{ Characteristic, n (\%) } & \multicolumn{2}{|l|}{ pCR } & \multicolumn{2}{|l|}{ non pCR } & \multirow[t]{3}{*}{$P$} \\
\hline & pTONO & pTON1-3 & pT1-4N0 & pT1-4N1-3 & \\
\hline & $n=25$ & $n=10$ & $n=40$ & $n=40$ & \\
\hline I & $1(4.0)$ & $1(10.0)$ & $2(5.0)$ & $0(0)$ & \\
\hline II & $3(12.0)$ & $2(20.0)$ & $0(0)$ & $3(7.5)$ & \\
\hline III & $14(56.0)$ & $6(60.0)$ & $34(85.0)$ & $31(77.5)$ & \\
\hline IVA & $1(4.0)$ & $1(10.0)$ & $0(0)$ & $1(2.5)$ & \\
\hline IVB & $6(24.0)$ & $0(0)$ & $4(10.0)$ & $5(12.5)$ & \\
\hline Recurrence & & & & & $0.0456^{\star}$ \\
\hline Presence & $5(20.0)$ & $4(40.0)$ & $15(37.5)$ & $22(55.0)$ & \\
\hline Absence & $20(80.0)$ & $6(60.0)$ & $25(62.5)$ & $18(45.0)$ & \\
\hline Prognosis & & & & & 0.3115 \\
\hline Alive & $22(88.0)$ & $6(60.0)$ & $27(67.5)$ & $24(60.0)$ & \\
\hline Dead with ESCC & $2(8.0)$ & $3(30.0)$ & $10(25.0)$ & $14(35.0)$ & \\
\hline Dead with other diseases & $1(4.0)$ & $1(10.0)$ & $3(7.5)$ & $2(5.0)$ & \\
\hline \multicolumn{6}{|c|}{ pCR pathological complete response, ESCC esophageal squamous cell carcinoma } \\
\hline * Statistically significant & & & & & \\
\hline
\end{tabular}

\section{The Survival Rate Of Patients With Non-pcr After Nacrt}

Comparison of the survival rates between the $\mathrm{PCR}$ and non-pCR groups revealed that $\mathrm{PCR}$ patients had significantly better OS, DSS, and RFS than non-pCR patients (Fig. 1a-1c). There were no significant differences in survival between $\mathrm{pTON}+, \mathrm{pT}+\mathrm{N} 0$, and $\mathrm{pT}+\mathrm{N}+$ subgroups, though survival among the $\mathrm{pT}+$ $\mathrm{N}+$ group was the poorest (Fig. 1d-1f).

Relationship between the SUV max $_{\text {ax }}$ reduction rate in the primary tumor and survival in the $\mathrm{pT}+\mathrm{N}+$ group.

We focused on the SUV $V_{\text {max }}$ reduction rate on FDG-PET measured in the primary tumors of 88 patients

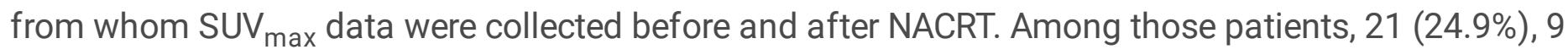
(10.2\%), 28 (31.8\%), and 30 (34.1\%) were categorized as pTONO, pTON+, pT + N0, or pT + N+, respectively. The median value of baseline SUV max $_{\text {ax }}$ and post-NACRT SUV max $_{\text {mere }} 14.8$ (2.9-42.0) and 3.2 (2.0-14.6), respectively. Based on the receiver operating characteristic (ROC) curve, the optimal cutoff value for the 
$S_{\text {SUV }}$ max reduction rate in the primary lesion for OS, DSS, and RFS was $60 \%$. We therefore performed Kaplan-Meier curve analyses using this cutoff value. Among all non-pCR patients, the 5-year OS and DSS were better in patients with a SUV $V_{\max }$ reduction rate of $60 \%$ or more than in those with a SUV $\max$ reduction rate of less than $60 \%$, though the differences were not statistically significant (Fig. 2a, 2b). However, 5-year RFS for patients with SUV max $_{\text {max }}$ reduction rates of $60 \%$ or more was significantly better than that for patients with $S_{U V} V_{\text {max }}$ reduction rates less than $60 \%$ (Fig. 2c).

We next divided the non-pCR patients into the three aforementioned subgroups and analyzed their prognosis using the optimal cutoff value for the SUV $\mathrm{max}_{\text {max }}$ reduction rate. In the pTON + subgroup, there were no differences in OS, DSS, and RFS between patients with SUV max $_{\text {max }}$ reduction rates of more than $60 \%$ and less than $60 \%$ (Fig. 3a-3c). In the pT + NO subgroup, there were also no differences in OS, DSS, or RFS between patients with SUV $V_{\text {max }}$ reduction rates greater than or less than $60 \%$ (Fig. 4a-4c). By contrast, in the $\mathrm{pT}+\mathrm{N}+$ subgroup, 5-year OS, DSS, and RFS among patients with SUV $\mathrm{max}_{\text {max }}$ reductions rates of $60 \%$ or

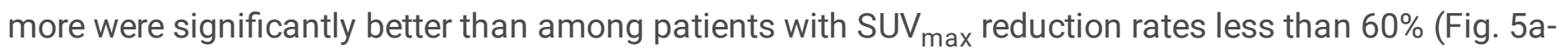
$5 c)$.

\section{Prognostic Analysis Of Os, Dss, And Rfs}

We performed univariate and multivariate survival analyses with the $\mathrm{p} T+\mathrm{N}+$ group (Table 2). Univariate analysis revealed that age $\left(\geq 65\right.$ or $<65$ ) and the SUV $V_{\text {max }}$ reduction rate in the primary tumor $(<60 \%$ or $\geq$ $60 \%$ ) were significant prognostic factors affecting 5 -year OS and DSS in the $\mathrm{pT}+\mathrm{N}+$ subgroup. Moreover, univariate analysis of 5-year RFS in the pT $+\mathrm{N}+$ group revealed that pathological depth of invasion (pT3-

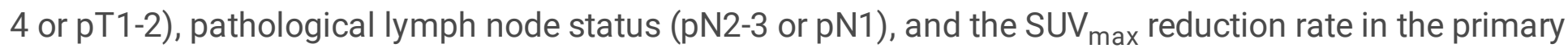
tumor ( $<60 \%$ or $\geq 60 \%$ ) were significant prognostic factors. Multivariate survival analysis of 5-year OS, DSS, and RFS in the $\mathrm{pT}+\mathrm{N}+$ group showed the $S U V_{\text {max }}$ reduction rate was an independent prognostic factor. It thus appears that in $\mathrm{pT}+\mathrm{N}+$ ESCC patients, the SUV $\mathrm{max}_{\text {ax }}$ reduction rate is clinically useful for predicting 5-year OS, DSS, and RFS. 
Table 2

Univariate and multivariate analyses of survival (Cox's proportional hazards regression models)

Factor

Age $(\geq 65 /<65)$

Gender (Male / Female)

Location (Middle, Lower / Upper)

Differentiation (Not poorly / poorly)

cT (T3-4 / T1-2)

CN (N2-3 / N1)

CM (M1(Lymph node) / M0)

pT (T3-4 / T1-2)

$\mathrm{pN}(\mathrm{N} 2-3 / \mathrm{N} 1)$

SUV $_{\max }$ reduction rate in the primary lesion $(<60 \% / \geq 60 \%)$

Factor

\section{Age $(\geq 65 /<65)$}

Gender (Male / Female)

Location ( Middle,Lower / Upper)

Differentiation (Not poorly / poorly)

cT (T3-4 / T1-2)

CN (N2-3 / N1)

cM (M1(Lymph node) / M0) os

Univariate

P-value

$0.0242 *$

0.9989

0.1605

0.6711

0.9991

0.7055

0.4804

0.2130

0.0965

$0.0007 *$

$8.3800(2.3893-29.3915)$

$0.0009 *$
P-value

$4.1216(1.1369-14.9423)$

0.0311 *

$\mathrm{HR}(95 \% \mathrm{Cl})$
Multivariate

$4.1216(1.1369-14.9423)$




\begin{tabular}{|c|c|c|c|}
\hline \multirow[t]{3}{*}{ Factor } & \multicolumn{3}{|l|}{ os } \\
\hline & \multirow{2}{*}{$\begin{array}{l}\text { Univariate } \\
\text { P-value }\end{array}$} & \multicolumn{2}{|l|}{ Multivariate } \\
\hline & & $\mathrm{HR}(95 \% \mathrm{Cl})$ & P-value \\
\hline pT (T3-4 / T1-2) & 0.4104 & & \\
\hline pN (N2-3 / N1) & 0.0969 & & \\
\hline $\begin{array}{l}\text { SUV }_{\max } \text { reduction rate in the primary lesion } \\
(<60 \% / \geq 60 \%)\end{array}$ & $0.0034^{*}$ & $7.6048(1.9819-29.1798)$ & $0.0031 *$ \\
\hline \multirow[t]{3}{*}{ Factor } & \multicolumn{3}{|l|}{ RFS } \\
\hline & Univariate & \multicolumn{2}{|l|}{ Multivariate } \\
\hline & P-value & $\mathrm{HR}(95 \% \mathrm{Cl})$ & P-value \\
\hline Age $(\geq 65 /<65)$ & 0.1947 & & \\
\hline Gender (Male / Female) & 0.2876 & & \\
\hline Location (Middle, Lower / Upper) & 0.0759 & & \\
\hline Differentiation (Not poorly / poorly) & 0.2910 & & \\
\hline cT (T3-4 / T1-2) & 0.9203 & & \\
\hline cN (N2-3 / N1) & 0.4032 & & \\
\hline cM (M1(Lymph node) / M0) & 0.7506 & & \\
\hline pT (T3-4 / T1-2) & $0.0312^{*}$ & $2.5857(0.8313-8.0423)$ & 0.1008 \\
\hline pN (N2-3 / N1) & $0.0330 *$ & $0.6968(0.1794-2.7072)$ & 0.6019 \\
\hline $\begin{array}{l}\text { SUV }_{\max } \text { reduction rate in the primary lesion } \\
(<60 \% / \geq 60 \%)\end{array}$ & $0.0006 *$ & $6.2962(1.4981-26.4625)$ & $0.0120 *$ \\
\hline \multicolumn{4}{|c|}{ OS overall survival, DSS disease-specific survival, RFS relapse-free survival, } \\
\hline \multicolumn{4}{|l|}{ HR hazard ratio, $\mathrm{Cl}$ confidence interval } \\
\hline * Statistically significant & & & \\
\hline
\end{tabular}

\section{Discussion}

In the present study, we demonstrated that there was no significant survival difference among patients with ESCC categorized as $\mathrm{pTON}+$, $\mathrm{pT}+\mathrm{N}$, or $\mathrm{pT}+\mathrm{N}+$ after treatment consisting of NACRT plus esophagectomy. Focusing on the SUV $V_{\max }$ reduction rate on FDG-PET in the primary tumor, we observed 
that patients with a SUV $\mathrm{max}_{\text {max }}$ reduction rate of less than $60 \%$ in the primary tumor after NACRT and categorized as $\mathrm{pT}+\mathrm{N}+$ after esophagectomy had the shortest 5-year OS, DSS, and RFS.

The development of multimodal therapies, such as chemotherapy, radiotherapy and surgery, have improved the prognosis of esophageal cancer patients. The ChemoRadiotherapy for Oesophageal cancer followed by Surgery Study (CROSS) trial found that NACRT plus surgery improved the survival rate among ESCC patients more than among esophageal adenocarcinoma patients. ${ }^{14}$ After CROSS trial, NACRT followed by surgery was widely accepted for treatment of esophageal cancer, especially ESCC. Many studies have consistently shown that pCR after NACRT is a significant prognostic factor predictive of favorable long-term outcomes in ESCC. ${ }^{15-17}$ Our finding that the 5-year OS rate in the pCR group was better than in non-pCR group is consistent with earlier reports.

While pCR patients obtained a benefit from NACRT followed by surgery, non-pCR patients did not. We therefore subdivided the non-pCR patients into $\mathrm{pTON}+, \mathrm{pT}+\mathrm{NO}$, and $\mathrm{pT}+\mathrm{N}+$ groups in effort to identify non-pCR patients who might benefit from NACRT followed by surgery. Several earlier reports have suggested that pathological N stage after NACRT is the most important prognostic factor in ESCC and that the number of pathological metastatic lymph nodes after NACRT was the strongest indicator of patient survival. ${ }^{18}$ Patients without pathological lymph node metastasis after NACRT followed by surgery were associated with a good prognosis, even when the CRT effect on the primary tumor was poor. ${ }^{19}$ We therefore expected that patients with pTON + and, especially, pT + NO would have better prognoses than those with $\mathrm{pT}+\mathrm{N}+$; however, no difference was found in their prognoses. Hatogai et al. showed that the $\mathrm{pN}$ factor based on the number of metastatic lymph nodes in the current staging system can predict a poor outcome among ESCC patients who have received NACT followed by surgery, but the pN factor was not an independent prognostic factor. ${ }^{20}$ They showed that the pathological tumor regression grade for main tumor and metastatic tumors in lymph nodes is predictive of prognosis in esophageal cancer patients who received NACT followed by surgery. ${ }^{20,21}$ In the present study, the survival curve for the pT + $\mathrm{N}+$ group was lowest among the three non-pCR groups, but there were no significant differences. For that reason, we focused on the SUV $V_{\text {max }}$ reduction rate on FDG-PET.

FDG-PET is useful for the diagnosis of metastasis before treatment and recurrence after treatment, and it can also be used to evaluate the response to neoadjuvant therapy. ${ }^{22,23} \mathrm{An}$ earlier study indicated that the SUV $_{\text {max }}$ reduction rate in the primary tumor may be a more significant predictor of CR after NACRT than the change in metabolic tumor volume (MTV) or total lesion glycolysis (TLG), two other parameters measured through FDG-PET examination. ${ }^{24}$ The optimal cutoff for the SUV max reduction in the primary tumor was $72 \%$ for pCR prediction after NACRT. ${ }^{24}$ Another recent report found that the SUV $\mathrm{max}_{\text {max }}$ reduction rate in the primary tumor is a valuable predictor of the pathological response to NACRT and survival. ${ }^{11}$ That report showed that, using a cut off of $75 \%$, the SUV $V_{\text {max }}$ reduction rate in the primary tumor can

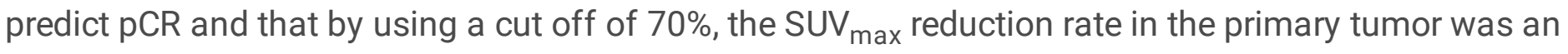
independent prognostic factor associated with better DSS. ${ }^{11}$ Those findings suggest that the degree of 
tumor metabolic response determined by preoperative FDG-PET is an important preoperative prognostic indicator for patients undergoing NACRT; moreover, the magnitude of the difference between the metabolic activities in primary tumors before and after NACRT is a useful guide for establishing postoperative therapy and postoperative surveillance programs. However, the cutoff values differ widely among studies, and the optimal cutoff value has not been determined. In the present study, ROC curve (data not shown) analysis showed that a SUV $\mathrm{max}_{\text {max }}$ reduction rate of $60 \%$ in the primary tumor was the optimal cutoff for predicting survival in non-pCR ESCC patients after NACRT. We suggest this cutoff value was lower than in previous reports because this analysis was limited to non-pCR patients among ESCC patients who underwent surgery after NACRT.

The results of this study show that patients with a SUV $\max$ reduction rate of less than $60 \%$ in the primary tumor after NACRT and categorized as $\mathrm{pT}+\mathrm{N}+$ after esophagectomy have an especially poor prognosis. We suggest that these patients should receive more powerful postoperative adjuvant therapy that differs from the preoperative chemotherapy regimens (e.g., immune checkpoint inhibitors). In addition, we also suggest that patients with a SUV $\mathrm{max}_{\text {max }}$ reduction rate of less than $60 \%$ in the primary tumor avoid surgery at that time and opt for stronger chemotherapy if $\mathrm{pT}+\mathrm{N}+$ after NACRT is clearly expected based on preoperative imaging examinations. It would then be advisable to consider surgery after determining the effectiveness of added chemotherapy.

We are aware that some limitations were inherent in this study. The retrospective nature of this study may have introduced selection bias. Because this study included only a small number of cases at a single institution, its impact is limited. In the future, a prospective research design in collaboration with other facilities will be required.

\section{Conclusions}

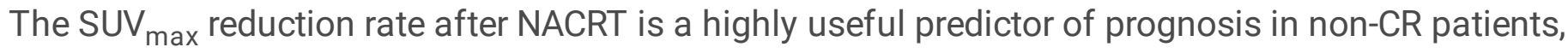
especially patients who are $\mathrm{pT}+\mathrm{N}+$ after NACRT and esophagectomy. Clinical non-CR patients with SUV $_{\max }$ reduction rates less than $60 \%$ on FDG-PET should be treated with additional adjuvant therapy before or after esophagectomy.

\section{Abbreviations}

$\mathrm{pCR}$

pathological complete response

NACRT

neoadjuvant chemoradiotherapy

NACT

neoadjuvant chemotherapy

ESCC

esophageal squamous cell carcinoma 
OS

overall survival

DSS

disease-specific survival

RFS

relapse-free survival

FDG-PET

${ }^{18}$ F-fluorodeoxyglucose-positron emission tomography

$\mathrm{SUV}_{\max }$

The maximum standardized uptake value

ECOG PS

Eastern Cooperative Oncology Group performance status

5 -FU

5-fluorouracil

CTCAE

Common Terminology Criteria for Adverse Events

CROSS

ChemoRadiotherapy for Oesophageal cancer followed by Surgery Study

\section{Declarations}

Ethics approval and consent to participate: This study was approved by the Ethics Committee of Akita University Graduate School of Medicine (No. 2406).

Consent for publication: All of the participants provided informed consent and signed human subject institutional review board consent forms.

Availability of data and materials: The datasets used and/or analysed during the current study are available from the corresponding author on reasonable request.

Competing interests: The authors declare that they have no competing interests.

Funding: S.M. is supported by the Kaken [17K10577].

Authors' contributions: Guarantors of integrity of entire study and study concepts: Nagaki Y, Motoyama S, and Minamiya Y. Study design or data acquisition or data analysis: Nagaki Y, Motoyama S, Sato Y, Wakita A, Fujita H, Sasaki Y, Imai K. Statistical analysis and manuscript editing: Nagaki Y. All authors read and approved the final manuscript.

Acknowledgements: We thank all members of our laboratories for technical assistance and helpful discussions. 


\section{References}

1. Rustgi AK, El-Serag HB. Esophageal carcinoma. N Engl J Med. 2014;371(26):2499-509.

2. Bancewicz J, Clark PI, Smith DB, et al. Medical Research Council, Oesophageal Cancer Working G. Surgical resection with or without preoperative chemotherapy in oesophageal cancer: A randomized controlled trial. Lancet. 2002;359:1727-33.

3. Kelsen DP, Winter KA, Gunderson LL, et al. Long-term results of RTOG trial 8911 (USA Intergroup 113):A random assignment trial comparison of chemotherapy followed by surgery compared with surgery alone for esophageal cancer. J Clin Oncol. 2007;25:3719-25.

4. Bosset JF, Gignoux M, Triboulet JP, et al. Chemoradiotherapy followed by surgery compared with surgery alone in squamous cell cancer of the esophagus. N Engl J Med. 1997;337:161-7.

5. Burmeister BH, Smithers BM, Gebski V, et al. Surgery alone versus chemoradiotherapy followed by surgery for resectable cancer of the oesophagus: A randomised controlled phase III trial. Lancet Oncol. 2005;6:659-68.

6. Tepper J, Krasna MJ, Niedzwiecki D, et al. Phase III trial of trimodality therapy with cisplatin, fluorouracil, radiotherapy, and surgery compared with surgery alone for esophageal cancer: CALGB 9781. J Clin Oncol. 2008;26:1086-92.

7. Ando $\mathrm{N}$, lizuka $\mathrm{T}$, Ide $\mathrm{H}$, et al. Surgery plus chemotherapy compared with surgery alone for localized squamous cell carcinoma of the thoracic esophagus: a Japan Clinical Oncology Group StudyJCOG9204. J Clin Oncol. 2003;21:4592-6.

8. Ando $\mathrm{N}$, Kato $\mathrm{H}$, Igaki $\mathrm{H}$, et al. A randomized trial comparing postoperative adjuvant chemotherapy with cisplatin and 5-fluorouracil versus preoperative chemotherapy for localized advanced squamous cell carcinoma of the thoracic esophagus (JCOG9907). Ann Surg Oncol. 2012;19:68-74.

9. Nakamura K, Kato K, Igaki H, et al Japan Esophageal Oncology Group/Japan Clinical Oncology Group. Three-arm phase III trial comparing cisplatin plus 5-FU (CF) versus docetaxel, cisplatin plus 5FU (DCF) versus radiotherapy with CF (CF-RT) as preoperative therapy for locally advanced esophageal cancer (JCOG1109, NExT study). Jpn J Clin Oncol. 2013;43(7):752-5.

10. Berger AC, Farma J, Scott WJ, et al. Complete response to neoadjuvant chemoradiotherapy in esophageal carcinoma is associated with significantly improved survival. J Clin Oncol. 2005;23:4330-7.

11. Hamai Y, Hihara J, Emi M, et al. Ability of fluorine-18fluorodeoxyglucose positron emission tomography to predict outcomes of neoadjuvant chemoradiotherapy followed by surgical treatment for esophageal squamous cell carcinoma. Ann Thorac Surg. 2016: 102:1132-9.

12. Rice TW, Patil DT, Blackstone EH. 8th edition AJCC/UICC staging of cancers of the esophagus and esophagogastric junction: application to clinical practice. Ann Cardiothorac Surg. 2017;6(2):119-30.

13. Sobin LH, Gospodarowicz MK, Wittekind Ch, editors. UICC International Union Against Cancer: TNM Classification of Malignant Tumours, 7th editon. Wiley-Blackwell, A John wiley \& Sons, Ltd. 2009. 
14. Shapiro J, van Lanschot JJB, Hulshof MCCM, et al. Neoadjuvant chemoradiotherapy plus surgery versus surgery alone for esophageal or junctional cancer (CROSS): long-term results of a randomized controlled trial. Lancet Oncol. 2015;16:1090-8.

15. Berger AC, Farma J, Scott WJ, et al. Complete response to neoadjuvant chemoradiotherapy in esophageal carcinoma is associated with significantly improved survival. $\mathrm{J}$ Clin Oncol. 2005;23:4330-7.

16. Scheer RV, Fakiris AJ, Johnstone PA. Quantifying the benefit of a pathologic complete response after neoadjuvant chemoradiotherapy in the treatment of esophageal cancer. Int J Radiat Oncol Biol Phys. 2011;80:996-1001.

17. Bollschweiler E, Hölscher AH, Metzger R. Histologic tumor type and the rate of complete response after neoadjuvant therapy for esophageal cancer. Future Oncol. 2010;6:25-35.

18. Akutsu $Y$, Shuto $K$, Kono $T$, et al. The number of pathologic lymph nodes involved is still a significant prognostic factor even after neoadjuvant chemoradiotherapy in esophageal squamous cell carcinoma. J Surg Oncol. 2012;105:756-60.

19. Okumura $H$, Uchikado $Y$, Matsumoto $M$, et al. Prognostic factors in esophageal squamous cell carcinoma patients treated with neoadjuvant chemoradiation therapy. Int J Clin Oncol. 2013;18:32934.

20. Hatogai K, Fujii S, Kojima T, et al. Prognostic significance of tumor regression grade for patients with esophageal squamous cell carcinoma after neoadjuvant chemotherapy followed by surgery. J Surg Oncol. 2016;113(4):390-6.

21. Kadota T, Hatogai $K$, Yano T,et al. Pathological tumor regression grade of metastatic tumors in lymph node predicts prognosis in esophageal cancer patients. Cancer Sci. 2018;109(6):2046-55.

22. Swisher SG, Erasmus J, Maish M, et al. 2-Fluoro-2-deoxy-D-glucose positron emission tomography imaging is predictive of pathologic response and survival after preoperative chemoradiation in patients with esophageal carcinoma. Cancer. 2004;101:1776-85.

23. Flamen $P$, van Cutsem $E$, Lerut $A$, et al. Positron emission tomography for assessment of the response to induction radiochemotherapy in locally advanced oesophageal cancer. Ann Oncol. 2002;13(3):361-8.

24. Park JS, Choi JY, Moon SH, et al. Response evaluation after neoadjuvant chemoradiation by positron emission tomography-computed tomography for esophageal squamous cell carcinoma. Cancer Res Treat. 2013;45:22-30.

\section{Figures}



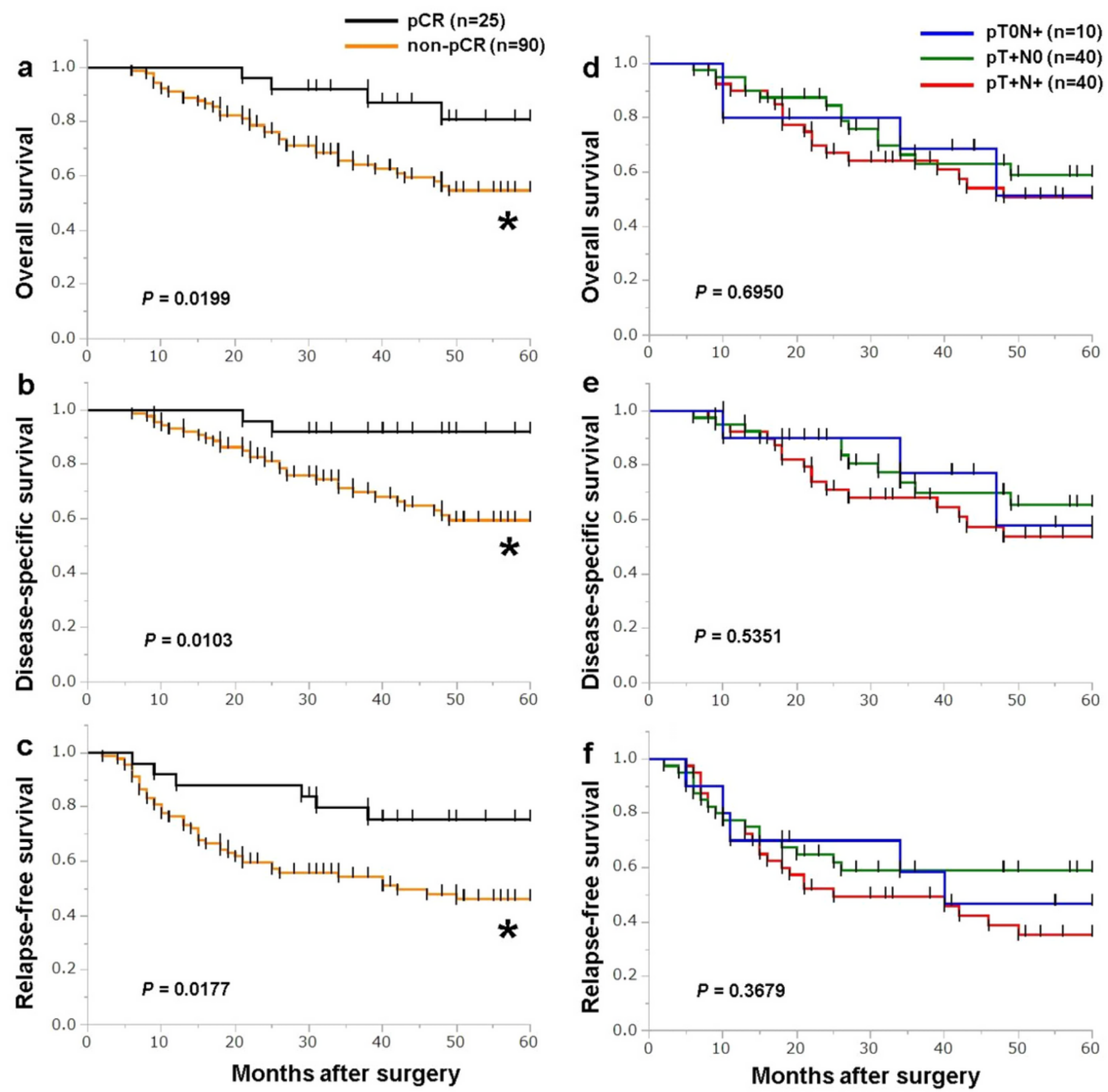

Figure 1

a-c, Kaplan-Meier survival curves showing OS (a), DSS (b), and RFS (c) among the pCR group (Black, $n=25$ ) and non-pCR group (Orange, $n=90)$. The log-rank test was used to compare the two groups. $d-f$, Kaplan-Meier survival curves showing OS (d), DSS (e), and RFS (f) among pTON+ group (Blue, $n=10$ ), $\mathrm{pT}+\mathrm{N} 0$ group (Green, $\mathrm{n}=40)$, and $\mathrm{pT}+\mathrm{N}+$ group $(\mathrm{Red}, \mathrm{n}=40)$. The log-rank test was used to compare the three groups. 

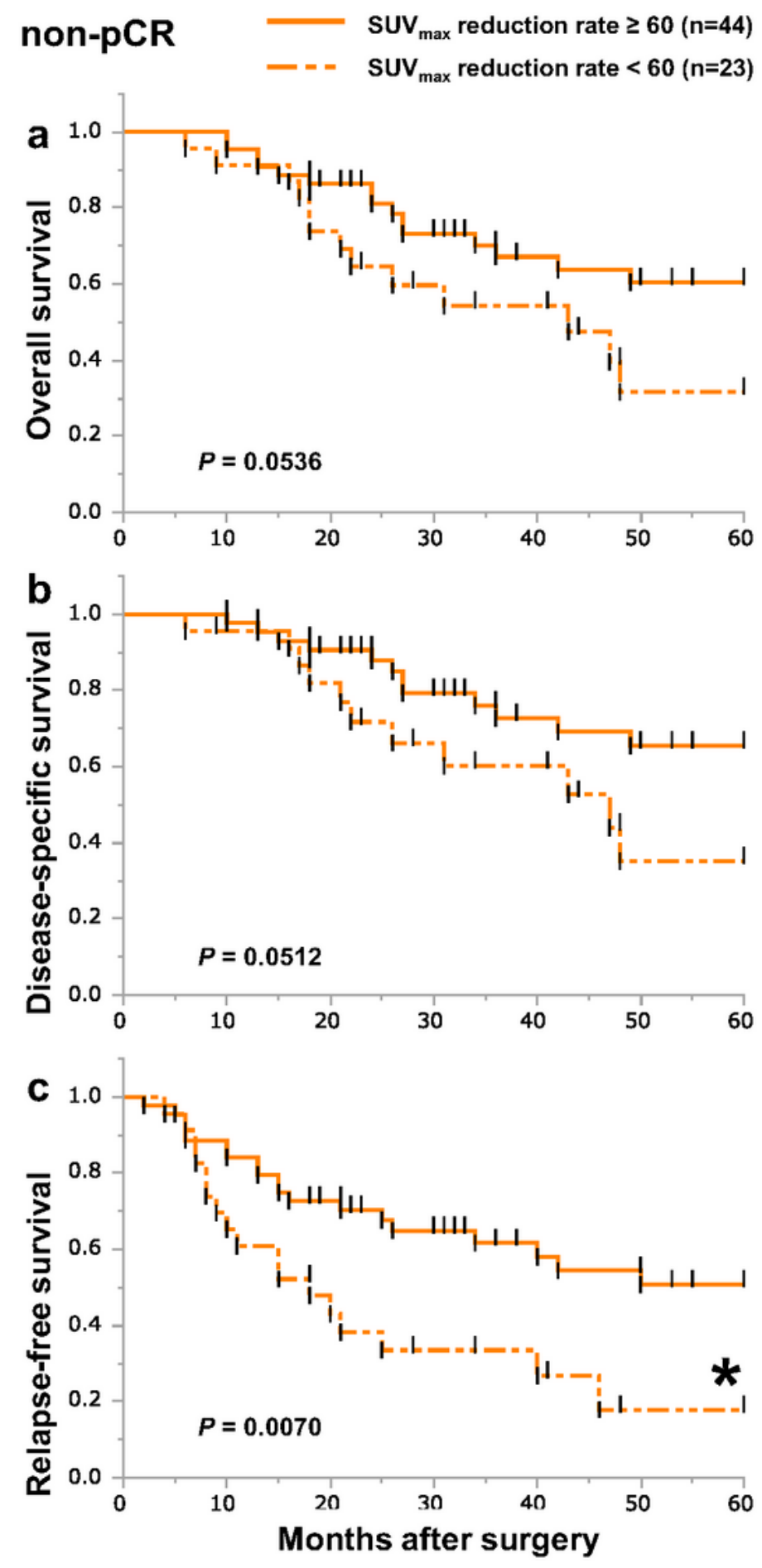

\section{Figure 2}

Kaplan-Meier survival curves showing OS (a), DSS (b), and RFS (c) according to the SUVmax reduction rate in the non-pCR group. Patients with SUVmax reduction rates of $60 \%$ or more in the primary tumor are indicated by solid lines $(n=44)$; those with SUVmax reduction rates of less than $60 \%$ in the primary tumor are indicated by dotted-dashed lines $(n=23)$. 

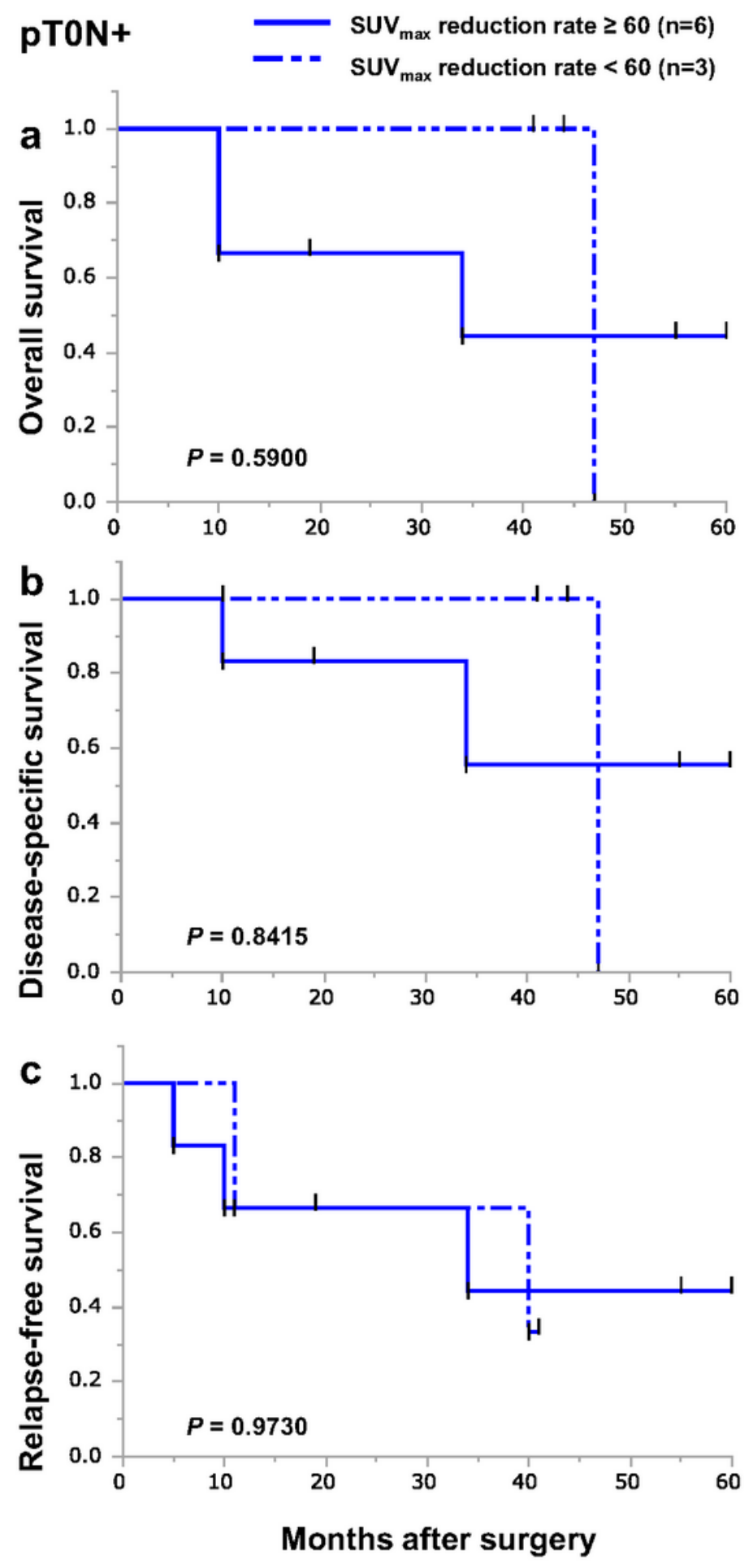

\section{Figure 3}

Kaplan-Meier survival curves showing OS (a), DSS (b), and RFS (c) according to the SUVmax reduction rate in the pTON+ group. Patients with SUVmax reduction rates of $60 \%$ or more in the primary tumor are indicated by solid lines $(n=6)$; those with SUVmax reduction rates of less than $60 \%$ in the primary tumor are indicated by dotted-dashed lines $(n=3)$. 

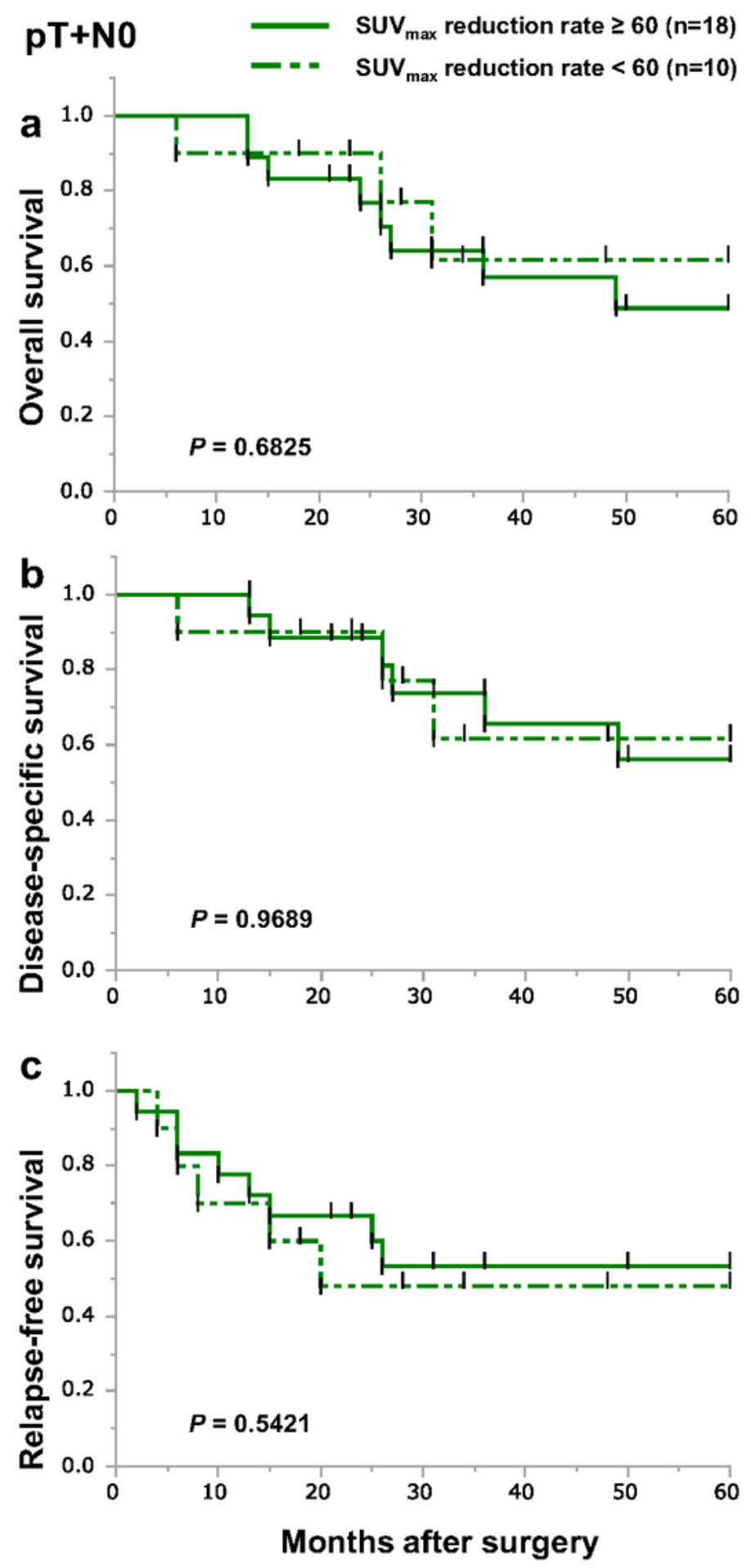

Figure 4

Kaplan-Meier survival curves showing OS (a), DSS (b), and RFS (c) according to the SUVmax reduction rate in the pT+NO group. Patients with SUVmax reduction rates of $60 \%$ or more in the primary tumor are indicated by solid lines $(n=18)$; those with SUVmax reduction rates of less than $60 \%$ in the primary tumor are indicated by dotted-dashed lines $(n=10)$. 

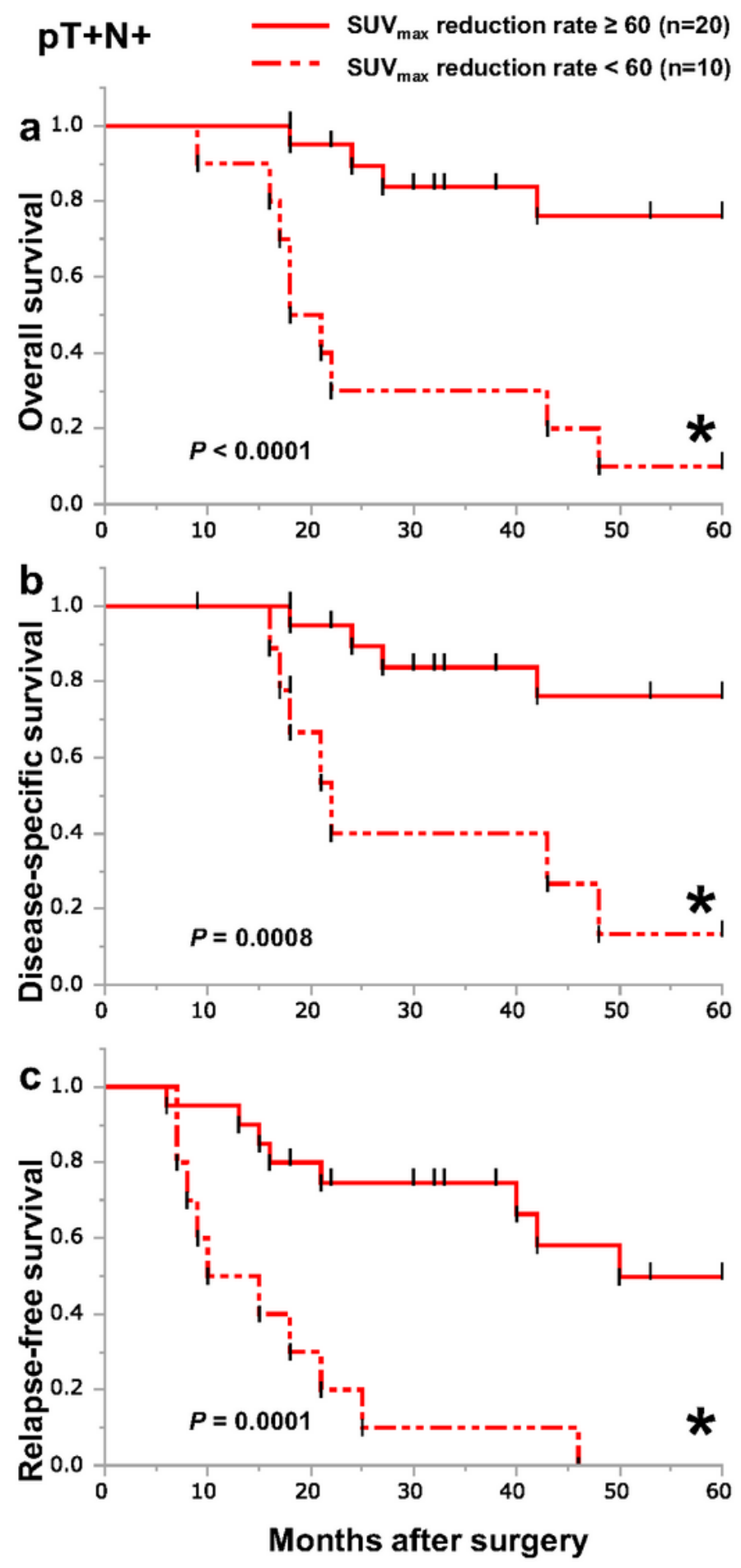

\section{Figure 5}

Kaplan-Meier survival curves showing OS (a), DSS (b), and RFS (c) according to the SUVmax reduction rate in the pT+N+ group. Patients with SUVmax reduction rates of $60 \%$ or more in the primary tumor are indicated by solid lines $(n=20)$; SUVmax reduction rates of less than $60 \%$ in primary tumor are indicated by dotted-dashed lines $(n=10)$. 